\title{
Ring Exchange and Correlated Fermions
}

\author{
Michel Roger \\ DSM-DRECAM, Service de Physique de l'Etat Condensé \\ Orme des Merisiers, CEA Saclay \\ 91191 Gif sur Yvette Cedex. France
}

\begin{abstract}
The concept of exchange in strongly-correlated fermions is reviewed with emphasis on the generalization of the Heisenberg pair exchange to higher order $n$-particle permutations. The "frustration" resulting from competing ferromagnetic three-spin exchange and antiferromagnetic two- and four-spin exchanges is illustrated on a twodimensional model system: solid ${ }^{3} \mathrm{He}$ films. Recent experimental results proving the presence of significant four-spin exchange interactions in the $\mathrm{CuO}_{2}$ plaquettes of high $T_{c}$ cuprates are reported.
\end{abstract}

Key words: Quantum spin systems, ${ }^{3} \mathrm{He}$, Cuprates.

\section{Historical introduction}

The concept of exchange interactions in almostlocalized correlated fermions first appeared in the pioneering papers of Heisenberg[1] and was formulated in a more general mathematical way by Dirac[2,3]. Although the early papers by Dirac already contain the general expression of the Hamiltonian in terms of n-particle permutations, no attention was paid, up to the sixties, to higher orders than the "pair-exchange" Heisenberg term. Thouless[4] was the first to point out that higher order exchanges as three- and four-spin cyclic permutations might be important in quantum solids like ${ }^{3} \mathrm{He}$. But only ten years later, striking experimental results on nuclear magnetism in the bcc phase of solid ${ }^{3} \mathrm{He}$, in the millikelvin range[5,6,7], were interpreted by Hetherington, Delrieu and Roger through a ring-exchange model with twothree- and four-particle interactions of comparable magnitude $[8,9,10]$. From general considerations put forward by Thouless, permutations of even parity (like three-particle cycles) induce ferromagnetism while odd permutations (pair and fourparticle exchanges) favor antiferromagnetism, and the striking phase diagram of bcc solid ${ }^{3} \mathrm{He}$ corresponds to a highly frustrated quantum-spin system with competing three- and four-spin permutations. During the last two decades a lot of progress has been accomplished in the investigation of solid ${ }^{3} \mathrm{He}$ films adsorbed on graphite, a simple model system exhibiting even more frustration since the frustrated nature of ring-exchange interactions is enhanced by the frustrated geometry of the triangular lattice. The conceptual beauty of solid ${ }^{3} \mathrm{He}$ lies in the fact that the system is simple enough (the pair interactions between ${ }^{3} \mathrm{He}$ atoms are mainly of hard core nature) to allow the calculation of exchange frequencies from first principles[11] and a quantitative comparison with 
experimental results.

The relevance of the multi-spin exchange concept is not restricted to the physics of nuclear ${ }^{3} \mathrm{He}$ spins. Delrieu[12] suggested that three-spin exchange might be dominant in the two-dimensional electron Wigner solid near the quasi-classical limit, and this has been corroborated through WKB calculations[13]. More recent Monte-Carlo simulations have proved that competing three and four-spin exchange interaction should occur near melting[14].

The first fourth-order $t / U$ expansion of the one-band Hubbard Hamiltonian in terms of fourspin interactions has been published in 1977 by Takahashi[15]. Soon after the discovery of high- $T_{c}$ superconductors, Roger and Delrieu[16] suggested, on the basis of an expansion of the three-band Hubbard model, that four-spin exchange might be significant in the $\mathrm{CuO}_{2}$ planes of cuprates. During the last ten years many experimental results have revealed the presence of four-spin exchange interactions in the $\mathrm{Cu}-\mathrm{O}$ plaquettes of cuprates $[17,18,19]$ and copper-based spin-ladder materials[20,21,22,23].

\section{Dirac formalism with illustration on the one-band Hubbard model.}

Dirac formalism is introduced in the framework of degenerate perturbation theory. The Hamiltonian is written $H=H_{0}+V$, where $H_{0}$ describes independent particles and $\mathrm{V}$ is a perturbation. The "unperturbed" degenerate ground-states for $\mathrm{N}$ distinguishable particles can be written as products of independent particle states:

$|\psi\rangle=\left|\alpha_{1}\right\rangle^{(1)}\left|\alpha_{2}\right\rangle^{(2)}\left|\alpha_{3}\right\rangle^{(3)} \cdots\left|\alpha_{N}\right\rangle^{(N)}$

which means that the particle numbered (i) is in a state $\left|\alpha_{i}\right\rangle$. Each of these states is itself a product of two kets corresponding to the orbital and spin variables respectively: $\left|\alpha_{i}\right\rangle=\left|R_{i}\right\rangle\left|\sigma_{i}\right\rangle$

$|\psi\rangle=\left|R_{1}\right\rangle^{(1)}\left|\sigma_{1}\right\rangle^{(1)} \cdots\left|R_{n}\right\rangle^{(N)}\left|\sigma_{N}\right\rangle^{(N)}$

Hence $|\psi\rangle=\left|\phi^{R}\right\rangle\left|\xi^{\sigma}\right\rangle$ appears as a product of an orbital wavefunction:

$\left|\phi^{R}\right\rangle=\left|R_{1}\right\rangle^{(1)}\left|R_{2}\right\rangle^{(2)} \cdots\left|R_{N}\right\rangle^{(N)}$ and a spin wavefunction:

$\left|\xi^{\sigma}\right\rangle=\left|\sigma_{1}\right\rangle^{(1)}\left|\sigma_{2}\right\rangle^{(2)} \cdots\left|\sigma_{N}\right\rangle^{(N)}$

Note that for the half-filled Hubbard model, $\left|R_{i}\right\rangle$ simply represents the site occupied by the particle (i). The permutation $\mathrm{P}$ of two particles can be expressed as a product of two operators:

$P=P^{R} P^{\sigma}$

$P^{R}$ acting on orbitals and $P^{\sigma}$ acting on spin variables. If the Hamiltonian does not depend explicitly on the spin, we can as a first step concentrate on the orbital part of the wave function and solve the eigenvalue problem:

$H\left|\phi^{R}\right\rangle=E\left|\phi^{R}\right\rangle$

for the orbital wavefunction $\left|\phi^{R}\right\rangle$ describing $\mathrm{N}$ distinguishable particles. The ground-state of the unperturbed part $H_{0}$ of the Hamiltonian is $\mathrm{N}$ ! fold degenerated and the corresponding subspace $\Omega_{0}$ is spanned by the N! states:

$P^{R}\left|\phi^{R}\right\rangle=\left|R_{\nu_{1}}\right\rangle^{(1)}\left|R_{\nu_{2}}\right\rangle^{(2)} \cdots\left|R_{\nu_{N}}\right\rangle^{(N)}$

where $\left\{\nu_{1}, \nu_{2}, \cdots, \nu_{N}\right\}$ represents a permutation $P$ of the $\mathrm{N}$ integers $\{1,2, \cdots, N\}$. We now apply degenerate perturbation theory $[25,26]$ to the perturbed Hamiltonian $H=H_{0}+V$. At first order, the splitting of the $\mathrm{N}$ ! degenerated energy levels is given by the eigenvalues of the Hamiltonian $V^{(1)}$ defined by its matrix elements:

$V_{a, b}^{(1)}=\left\langle\phi^{R}\left|P_{a}^{R} V P_{b}^{R}\right| \phi^{R}\right\rangle$

where $P_{a}^{R}$ and $P_{b}^{R}$ are two permutations of the symmetric group $S_{N}$. Since $V$ is invariant with respect to any permutation, we can write:

$V_{a, b}^{(1)}=\left\langle\phi^{R}\left|V P_{a}^{R} P_{b}^{R}\right| \phi^{R}\right\rangle=\left\langle\phi^{R}\left|V P^{R}\right| \phi^{R}\right\rangle=V_{P}^{(1)}$

where $P=P_{a}^{R} P_{b}^{R}$, and the eigenvalue problem, restricted to the subspace $\Omega_{0}$ can be formally represented by the Hamiltonian:

$H^{(1)}=-\sum_{P^{R} \in S_{N}} V_{P}^{(1)} P^{R}$

where the summation runs over permutations $P^{R}$ of the symmetric group $S_{N}$. This result extends 
straightforwardly to higher order degenerate perturbation theory: the higher orders are expressed in terms powers of $V$ and projection operator $P_{0}$ on $\Omega_{0}[25,26]$, and these operators commute with permutation operators. Hence, at arbitrary order in degenerate perturbation theory, we can write:

$H \approx-\sum_{P^{R} \in S_{N}} V_{P} P^{R}$

We now have to introduce the spin degrees of freedom an express that the global wavefunction is completely antisymmetric. As a general result from Group theory a completely antisymmetric wave function can be expressed by the following bilinear expression[27]:

$|\psi\rangle=\sum_{\lambda, \mu} c_{\lambda, \mu}\left|\phi_{\lambda}^{R}\right\rangle\left|\xi_{\mu}^{\sigma}\right\rangle$

where $\left|\phi_{\lambda}^{R}\right\rangle$ represents a linear combination of different permutations $P_{a}^{R}\left|\phi^{R}\right\rangle$ corresponding to a given irreducible representation of the symmetry group schematized by a Young diagram, while $\left|\xi_{\mu}^{\sigma}\right\rangle$ represents a linear combination of permutation $P_{\bar{a}}^{\sigma}\left|\xi^{\sigma}\right\rangle$ corresponding to the representation associated with the "complementary" Young diagram, obtained by exchanging the lines and the columns. For spin-1/2, a complete antisymmetrisation of the spins cannot be realised over more than 2 variables, hence the corresponding Young diagrams have at most two lines and each diagram corresponds to a given value of the total spin $S$. It is then possible to establish a correspondence between the expression [Eq. (10)] of the Hamiltonian acting only on the orbital variables with an equivalent Hamiltonian acting only on the spin variables. Expressing the antisymmetry of the wave function:

$P|\psi\rangle=(-1)^{p}|\psi\rangle$

where $p$ is the parity of the permutation, we can write:

$P^{R} P^{\sigma}|\psi\rangle=(-1)^{p}|\psi\rangle$

and multiplying to the left by $\left(P^{R}\right)^{-1}$ :

$P^{\sigma}|\psi\rangle=(-1)^{p}\left(P^{R}\right)^{-1}|\psi\rangle$
Taking into account that in Eq. (10) $P^{R}$ and the inverse permutation $\left(P^{R}\right)^{-1}$ appear with the same weight $V_{P}$, the Hamiltonian is written equivalently in spin space:

$H \approx-\sum_{P^{\sigma} \in S_{N}}(-1)^{p} V_{P} P^{\sigma}$

where the sum is over all permutations of the symmetric group $S_{N}$ acting on spin variables.

Large $U$ expansions of the one-band Hubbard model[15] have generally been performed within the framework of second quantization (i.e working on the total antisymmetric wavefunction). Dirac formalism allows a simpler and more physical derivation. We start with $\mathrm{N}$ distinguishable particles on $\mathrm{N}$ discrete sites, each site containing zero, one or two particles. There is an onsite Coulomb energy $U$ for putting two particles on the same site, and each particle has a probability $t$ to hop from one site to the nearest neighbor. The Hamiltonian does not depend explicitly on the spin variables.

In perturbation theory, we start from the infinite $U$ limit: each site is occupied by one particle. Since the particles are considered as distinguishable, the ground state is N! fold degenerated. We apply degenerate perturbation theory to express the energy splitting as an expansion in $t / U$ when $U$ is large but finite[25,26]. Up to fourth order, the effective Hamiltonian restricted to the subspace $\Omega_{0}$ spanned by the $\mathrm{N}$ ! fold degenerated ground states is written[15,25,26]:

$P_{0} V S V P_{0}+\left[P_{0} V S V S V S V P_{0}-P_{0} V S^{2} V P_{0} V S V P_{0}\right]$

where $P_{0}$ is the projection operator onto the subspace $\Omega_{0}$ and $S=\left(1-P_{0}\right) /\left(E_{0}-H_{0}\right)$. This expression can be expanded in term of two, three and four-particle permutations $P^{R}$ acting on coordinates:

$$
\begin{array}{r}
H=-J_{1} \sum_{<i, j>}^{(1)} P_{i j}^{R}-J_{2} \sum_{<i, k>}^{(2)} P_{i k}^{R}-J_{3} \sum_{<i, l>}^{(3)} P_{i l}^{R}- \\
J_{T} \sum_{<i, j, k>}\left[P_{i j k}^{R}+\left(P_{i j k}^{R}\right)^{-1}\right]- \\
K \sum_{<i, j, k, l>}\left[P_{i j k l}^{R}+\left(P_{i j k l}^{R}\right)^{-1}\right]
\end{array}
$$




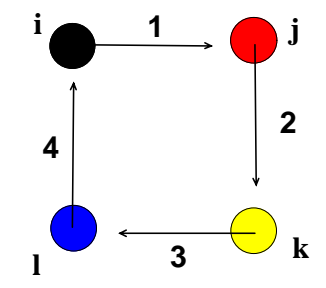

Fig. 1. Evaluation of the four-particle cyclic permutation frequency. Only the term $P_{0} V S V S V S V P_{0}$ in the fourth order expansion contribute to the cyclic exchange of the four particle coordinates $i \rightarrow j, j \rightarrow k, k \rightarrow l$ and $l \rightarrow i$. Each of these four particle hops is accomplished by one of the four $V$ in the previous term and at each step a factor $-t_{\alpha \beta} /(\nu U)$ where $\nu$ is the number of doubly occupied sites, in the corresponding intermediate virtual state, has to be taken into account (this factor arises from the operator $S$ ). There are $4 !=24$ terms depending on the order in which the four hops 1, 2, 3, 4, are successively realised. Eight of them [corresponding to the sequences (1324), (1342), (3124), (3142), (2413), (4213), (2431), (4231)] contribute to a factor $-t^{4} /\left(2 U^{3}\right)$, the sixteen others contribute to $-t^{4} / U^{3}$. The total contribution is then $-20 t^{4} / U^{3}$.

with: $J_{1}=2\left(t^{2} / U\right)\left(1+4 t^{2} / U\right), J_{2}=12 t^{4} / U^{3}$, $J_{3}=2 t^{4} / U^{3}, J_{T}=10 t^{4} / U^{3}$ and $K=20 t^{4} / U^{3}$ for the square lattice. The sums in the first line are on first, second and third neighbor pairs respectively. The sum run over triangles in the second line and over square plaquettes in the last line. As a typical example, the Fig. 1 illustrates the evaluation of the four-particle cyclic permutation term.

Since cyclic two and four-particle permutations are of odd parity, while three-particle permutations are even, we obtain the corresponding exchange Hamiltonian, with permutation operators $P^{\sigma}$ acting on spin variables::

$$
\begin{aligned}
H_{e x}=J_{1} \sum_{<i, j>}^{(1)} P_{i j}^{\sigma}+J_{2} \sum_{<i, k>}^{(2)} P_{i k}^{\sigma}+J_{3} \sum_{<i, l>}^{(3)} P_{i l}^{\sigma}- \\
J_{T} \sum_{<i, j, k>}\left[P_{i j k}^{\sigma}+\left(P_{i j k}^{\sigma}\right)^{-1}\right]+ \\
K \sum_{<i, j, k, l>}\left[P_{i j k l}^{\sigma}+\left(P_{i j k l}^{\sigma}\right)^{-1}\right]
\end{aligned}
$$

Using the identity: $P_{i, j}^{\sigma}=\left(1+\sigma_{i} \cdot \sigma_{j}\right) / 2$ and the decomposition of any permutation in a product of transpositions, it is easy to check that this Eq. 16 is identical to the result given by Takahashi. However our previous expression clearly separate the ferromagnetic contributions arising from three-particle exchange and the antiferromagnetic ones coming from pair- and four-particle exchange. The formulation in terms of permutation operators is also generally more convenient for the calculation of the thermodynamic properties of a n-particle exchange Hamiltonian.

\section{Cyclic exchange in solid ${ }^{3} \mathrm{He}$ and in the Wigner Solid from first principles}

Dirac formalism has been generalized by Thouless for the exchange-problem in solid ${ }^{3} \mathrm{He}[4]$ and by Herring for many-electron systems [24]. This has been applied later to ab-initio calculations of multi-spin exchange frequencies in solid ${ }^{3} \mathrm{He}$ and in the two-dimensional Wigner-Solid. A pioneering multidimensional WKB calculation of the exchange frequencies has been applied to the twodimensional Wigner solid near the quasi-classical limit[13]. It has proved the conjecture by Delrieu that three-particle exchange should dominate in that regime. Although, at physical densities, solid ${ }^{3} \mathrm{He}$ is far from the quasi-classical regime, the same WKB approach was extrapolated with qualitative results concerning the hierarchy of various cyclicexchange frequencies.

With the rapid increase of the performances of computers, ab-initio calculations of ring-exchange frequencies through Path Integral Monte Carlo (PIMC) became possible. In contrast to WKB evaluation they are relevant to the whole range of physical densities, including solid phases near melting where quantum fluctuations are large. The exchange frequencies for bcc solid ${ }^{3} \mathrm{He}[11]$ near melting, are in excellent agreement with the experimental thermodynamic properties, which can be inferred through exact high-temperature series expansions.

Although the long-range Coulomb potential is completely different from the hard core potential between Helium atoms, WKB calculations near the classical limit[13,28] and PIMC in the whole density range[14] give striking analogies between solid ${ }^{3} \mathrm{He}$ films and the electron Wigner 
solid, on the same triangular lattice. In both systems, three-particle exchange dominates near the quasi-classical limit, leading to ferromagnetism, while near melting, antiferromagnetic two- and four-particle exchange compete with ferromagnetic three-particle exchange leading to highly frustrated antiferromagnetic systems.

Close to melting, both systems might be prototypes of two-dimensional "spin liquids" [29] with no long range order at $\mathrm{T}=0$. Experiments on the magnetism of the Wigner Solid are now at the very beginning[30], but the nuclear magnetism of solid ${ }^{3}$ He films has now been extensively studied.

\section{Two-dimensional solid ${ }^{3}$ He: a model system with tunable frustration.}

Exfoliated graphite offers large $(10 \times 10 \mathrm{~nm})$ flat crystallites with a strong adsorption potential for He. Up to two solid He films can be adsorbed on this substrate, within a wide density-range. The magnetism of 2D helium films has been thoroughly studied, down to $10-100 \mu \mathrm{K}$ in:

(i) submonolayer solid ${ }^{3}$ He films on graphite[31]

(ii) solid ${ }^{3} \mathrm{He}$ films on graphite coated by a high density ${ }^{3} \mathrm{He}[32,33,34,35,36]$ or ${ }^{4} \mathrm{He}[38,39]$ layer or by a HD bilayer[37].

The main difference between (i) and (ii) is that, due to a stronger adsorption potential, the first layer is closer to the pure $2 \mathrm{D}$ case. At the same density exchange frequencies in the first layer are 10 times lower than in the second layer where ${ }^{3} \mathrm{He}$ atom permutations are made easier through excursion in the third dimension. However the same general trends are observed:

- A low-density commensurate solid phase is stabilized by the graphite potential in case (i) and by the periodic potential of the first layer in case (ii). Its susceptibility has an antiferromagnetic character with a negative Curie-Weiss constant. Both susceptibility and specific heat present anomalous deviations with respect to the usual asymptotic behavior at high temperature (Curie Weiss law for the susceptibility and $\mathrm{T}^{-2}$ behavior for the specific heat). These features are due to the frustrated nature of the system and can be quantitatively fit through the ring-exchange model[36]. Down to $10 \mu \mathrm{K}$ no long-range ordering has been detected, which seems to confirm the presence of a spin-liquid state. An ultra-low temperature specific-heat measurement[35] and susceptibility measurements[38,39] put a higher limit of $10-100 \mu \mathrm{K}$ on a possible gap in the excitation spectrum. Hence solid ${ }^{3} \mathrm{He}$ films could be the first experimental realization of a gapless spin liquid.

- At higher densities, an incommensurate solid phase is observed. The susceptibility has a positive Curie Weiss constant, but the unusual features of the specific heat indicate that we have a "frustrated ferromagnetic" phase with relatively larger three-spin exchange but significant competing four-particle exchange[36]. The frustration is easily "tunable" in the sense that when the density is further increased, three-spin exchange dominates leading to a more conventional ferromagnet.

Some anomalies in the specific heat are present only in the commensurate phases. A very interesting interpretation has been recently proposed by H. Fukuyama [31], in terms of "ground-state vacancies" surrounded by magnetic polarons. This exciting idea established a close link with strongly interacting almost-localized electron systems close to a Mott-Hubbard transition (e.g the physics of cuprates!).

\section{Four-spin exchange in high- $\mathbf{T}_{c}$ Cuprates}

In the insulating phases of Cuprates, the first experimental results in contradiction with a pure Heisenberg model were the anomalously broad multi-magnon Raman spectrum[17] and the infrared optical absorption by phonon assisted multimagnon excitations [40] in $\mathrm{La}_{2} \mathrm{CuO}_{4}$. Both results can be understood with the occurrence of four-spin exchange[17,20]. However the key experiments which really invalidates the pure Heisenberg model is the inelastic neutron study of the magnon spectrum near the Brillouin zone[18]. It is in agreement with the presence of ferromagnetic three-spin exchange terms $J_{T}$ as those appearing 
in the expansion of the one-band Hubbard model (Eq. 16). However, due to accidental cancellations occurring for the Néel phase of the square lattice, the linear spin-wave spectrum is insensitive to the presence of four-spin exchange operators[19]. An excellent way to probe directly the presence of four-spin terms is the study of spin-spin correlations in the paramagnetic phase. These correlation have been recently measured through diffuse magnetic scattering of polarized neutrons in $\mathrm{La}_{2} \mathrm{CuO}_{4}$ at $350 K<T<500 K[19]$. The results are compared to predictions from high-temperature series expansions of the Hamiltonian. They prove definitively the presence of four-spin interaction with a ratio $K / J_{1}$ of about $20 \%$.

The same $\mathrm{Cu}-\mathrm{O}$ plaquettes are present in copper based quasi-1D spin ladder materials $\mathrm{Sr}_{1} 4 \mathrm{Cu}_{24} \mathrm{O}_{41}$ and $\mathrm{La}_{6} \mathrm{Ca}_{8} \mathrm{Cu}_{24} \mathrm{O}_{41}$, with approximately the same $\mathrm{Cu}-\mathrm{O}$ bonds and distances as in $\mathrm{La}_{2} \mathrm{CuO}_{4}$. The first neighbor pair exchanges along rungs and legs are expected to be roughly equal $J_{\text {rung }} \approx J_{\text {leg }}=J$. In that case the theory predict a gap $\Delta \approx 0.5 J$ in the excitation spectrum. The experimental value of the gap is substantially lower: $\Delta_{\exp } \approx 0.35 J[21,22]$. A relatively small four-spin exchange $K / J \approx 20 \%$ accounts for the decrease of the gap[23]. This effect is related to the frustration due to four-spin exchange, which increases the density of low lying eigenstates.

The concept of multi-particle exchange, pioneered by Dirac at the beginning of the 20th century, now appears as essential in the physics of strongly-correlated fermion systems which have raised considerable interest during the last decades.

References

[1] W. Heisenberg, Z. Phys, 38 (1926) 411 ; ibid 49 (1928) 619.

[2] P. A. M. Dirac, Proc. Roy. Soc. A 123 (1929) 714.

[3] P. A. M Dirac, The Principles of Quantum Mechanics (Clarendon, Oxford) Ch. IX, (1947).

[4] D.J. Thouless, Proc. Phys. Soc. London 86 (1965) 893.

[5] R.B. Kummer et al, Phys. Rev. Lett. 34 (1975) 517; E.D Adams, J.M. Delrieu. A. Landesman, J. Phys. Lett. 39 (1978) L190.
[6] H. Godfrin et al., Phys. Rev. Lett. 44 (1980) 1695.

[7] D.D. Osheroff, M.C. Cross, D.E. Fisher, Phys. Rev. Lett. 44 (1980) 792.

[8] J.H. Hetherington, Phys. Rev. Lett. 35 (1975) 1442.

[9] J.M. Delrieu et al. J. Low. Temp. Phys. 40 (1980) 71.

[10] M. Roger, J.M. Delrieu, J.H. Hetherington, Rev. Mod. Phys. 55 (1983) 1.

[11] D.M. Ceperley, G. Jacucci, Phys. Rev. Lett. 58 (1987) 1648.

[12] J.M. Delrieu, private communication (1980).

[13] M. Roger, Phys. Rev. B30 (1984) 6432.

[14] B. Bernu, L. Candido, D.M. Ceperley, Phys. Rev. Lett. 86 (2001) 870 .

[15] M. Takahashi, J. Phys. C. 10 (1977) 1289.

[16] M. Roger, J.M. Delrieu, Phys. Rev. B39 (1989) 2299.

[17] S. Sugai et al. Phys. Rev. B42 (1990) 1405.

[18] R. Coldea et al. Phys. Rev. Lett. 86 (2001) 5377.

[19] A. Toader et al. to be published

[20] J. Lorenzana, J. Eroles, S. Sorella, Phys. Rev. Lett. 83 (1999) 5122.

[21] Imai et al. Phys. Rev. Lett. 81 (1998) 220.

[22] Eccleston et al. Phys. Rev. Lett. 81 (1998) 1702.

[23] S. Brehmer et al. Phys. Rev. B 60 (1999) 329.

[24] C. Herring in Magnetism Vol. II B ed. Rado and Suhl, (Academic Press, London) Ch. I, (1966).

[25] T. Kato, Progress of Theoretical Physics IV, 514 (1949); ibid V (1950) 207.

[26] C. Bloch, Nuclear Physics 6 (1958) 329.

[27] L. Landau, E. Lifchitz, Quantum Mechanics, (Pergamon Press, London) Ch. IX, (1959).

[28] M. Katano, D.S. Hirashima, Phys. Rev. B 62 (2000) 2573.

[29] G. Misguich et al., Phys. Rev. Lett. 81 (1998) 1098.

[30] T. Okamoto, S. Kawaji, Phys. Rev. B 57 (1998) 9097.

[31] M. Morishita, H. Nagatani, H. Fukuyama, Phys. Rev. B 65 (2002) 104524.

[32] The density of the completed first ${ }^{3} \mathrm{He}$ layer is so high that its magnetism is negligible.

[33] D.S. Greywall, Phys. Rev. B 41 (1990) 1842.

[34] M. Siquera et al., Phys. Rev. Lett. 78 (1997) 2600.

[35] K. Ishida et al., Phys. Rev. Lett. 79 (1997) 3451.

[36] M. Roger et al., Phys. Rev. Lett. 80 (1998) 1308.

[37] H. Ikegami et al., Phys. Rev. Lett. 85 (2000) 5146.

[38] E. Collin et al. Phys. Rev. Lett. 86 (2001) 2447. 
[39] R. Masutomi, Y. Karaki, H. Ishimoto, Phys. Rev. Lett. 92 (2004) 025301

[40] J.D. Perkins et al., Phys. Rev. Lett. 71 (1993) 1621. 